\title{
PRODUÇÃO DE MUDAS DE SABIÁ Mimosa caesalpiniaefolia Benth NODULADAS E MICORRIZADAS EM DIFERENTES SUBSTRATOS
}

\author{
S. R. L. TAVARES ${ }^{1 *}$, A. A. FRANCO ${ }^{2}$ e E. M. R. SILVA ${ }^{2}$ \\ ${ }^{1}$ Empresa Brasileira de Pesquisa Agropecuária (EMBRAPA SOLOS) \\ ${ }^{2}$ Empresa Brasileira de Pesquisa Agropecuária (EMBRAPA AGROBIOLOGIA) \\ silvio.tavares@embrapa.br*
}

Artigo submetido em dezembro/2015 e aceito em setembro/2016

DOI: $10.15628 /$ holos.2016.3933

\section{RESUMO}

Para avaliar os efeitos da inoculação com bradyrhizobium e fungos micorrízicos arbusculares em dois substratos usados para a formação de mudas de sabiá (Mimosa caesalpiniaefolia Benth), no crescimento, nodulação, colonização micorrízica e teores e acúmulos de macro e micronutrientes, foi realizado um experimento em casa de vegetação na Embrapa Agrobiologia, utilizando um delineamento experimental inteiramente casualizado, em esquema fatorial completo $4 \times 2$ com 5 repetições. Os tratamentos foram: Testemunha sem inoculação; inoculação com rizóbio; inoculação com micorriza; inoculação com rizóbio + micorriza e dois substratos com diferentes conteúdos de composto orgânico em sua composição. Avaliaram-se os parâmetros de crescimento, nutrição, nodulação e colonização das mudas aos 79 dias após o plantio. As mudas colonizadas isoladas $(R)$ e/ou em conjunto $(R+M)$ beneficiaram a formação das mudas do sabiá em todos os parâmetros de crescimento estudados em relação aos outros tratamentos microbiológicos (Test. e M); aumentaram os teores de $\mathrm{N}$ e o acúmulo de $\mathrm{N}, \mathrm{K}, \mathrm{Mg}, \mathrm{Cu}, \mathrm{Mn}$ e Fe. A inoculação com micorriza também estimulou a nodulação no sabiá. Os resultados finais indicam que, em relação as variáveis estudadas, deve-se optar pelo substrato com menor teor de composto orgânico para a produção de mudas dessa espécie que é muito indicada para utilização em campo pelas unidades de pesquisas da Embrapa.

PALAVRAS-CHAVE: Mudas florestais, FBN, simbiose, RAD, nutrição vegetal.

\section{PRODUCTION OF SABIÁ SEEDLINGS (Mimosa caesalpiniaefolia Benth) IN DIFFERENT SUBSTRATES WITH RHIZOBIUM AND MYCORRHIZA}

\begin{abstract}
The effects of inoculation of bradyrhizobium and arbuscular mycorrhizal fungus and two substrates used to grow seedlings of sabiá (Mimosa caesalpiniaefolia Benth) were evaluated by measuring the growth, nodulation, colonization of mycorrhizal fungus, plant growth and accumulation of nutrients. The experiment were accomplished at Embrapa Agrobiologia, with a complete factorial (4 $\times 2$, with 5 replications) in a complete randomized sampling model design. The treatments were: Control, inoculation with rhizobium; inoculation with Mycorrhiza; inoculation with rhizobium + Mycorrhiza; and two substrates with different contents of organic material. Growth, nutrition, nodulation and
\end{abstract}

colonization parameters in the seedlings were evaluated after 79 days for sabiá after planning. The isolated colonized seedlings $(R)$ and / or together $(R+M)$ received thrush the formation of seedlings in all growth parameters studied in relation to other microbiological treatments (Test and $\mathrm{M}$.); increased $\mathrm{N}$ content and accumulation of $\mathrm{N}, \mathrm{K}, \mathrm{Mg}, \mathrm{Cu}, \mathrm{Mn}$ and Fe. Inoculation with mycorrhiza also stimulated nodulation in thrush. The final results indicate that, for the variables, you should opt for the substrate with less organic compound content for the production of seedlings of this species which is very suitable for field use by Embrapa research units.

KEYWORDS: Grow seedlings, biological nitrogen fixation, symbiosis, recovery of degraded area, plant nutrition. 


\section{INTRODUÇÃO}

Segundo o Banco Mundial, os solos agrícolas do mundo vêm se degradando a uma taxa de $0,1 \%$ ao ano, dados que vão de encontro dos estabelecidos pela Organização das Nações Unidas para a Alimentação e Agricultura (FAO), que aponta a perda de cinco milhões de hectares de terras aráveis por ano devido a más práticas agrícolas, secas e pressão populacional, além de inúmeras ações antrópicas da exploração inadequada dos recursos naturais englobando o compartimento solo (CAPECHE, et al., 2014).

Desse panorama surge a necessidade de se produzirem mudas de essências florestais para suprir a demanda de programas de recomposição da vegetação nessas áreas (CARVALHO FILHO et al., 2003). Geralmente, a revegetação e/ou a incorporação dessas áreas ao processo produtivo é feita com elevado custo financeiro e energético, marginalizando cada vez mais as extensas áreas degradadas e a grande maioria dos agricultores que compõem a esfera mais pobre da economia rural brasileira.

O desenvolvimento de protocolos e estratégias para a produção de mudas com qualidade, em menor tempo e em condições acessíveis é muito importante. Dessa maneira, estudos envolvendo substratos alternativos e recipientes adequados para baratear os custos de produção e a inoculação das mudas com fungos micorrízicos arbusculares e demais microrganismos que favorecem o estabelecimento das mudas nas áreas degradadas são de grande valia (SOUZA et al., 2009).

O sucesso do plantio em grande escala de espécies florestais está associado à produção de mudas de boa qualidade, sua sobrevivência e estabelecimento no campo (SCHIAVO et al., 2009). Um sistema interativo tripartite (leguminosa - fungo micorrízico - rizóbio) é capaz de prover melhores condições fisiológicas às mudas de leguminosas do sistema, de modo que a dupla simbiose estabelecida permite a muda capturar com melhor eficiência os nutrientes, sobretudo o nitrogênio e o fósforo (SOUZA \& SILVA, 1996), respondendo diretamente sob o desenvolvimento do sistema radicular, o que reflete no crescimento e desenvolvimento da parte aérea da planta, assegurando às mudas uma maior tolerância a características físico-químicas inadequadas do solo, agregando maiores chances de estabelecimento e desenvolvimento em campo (SOUCHIE et al., 2005, apud MENDES, 2010).

Neste contexto, o Comunicado Técnico $\mathrm{n}^{\circ} 9$ sobre revegetação de solos degradados da Embrapa Agrobiologia, indicava a utilização de um substrato que consistia de uma mistura contendo, em volume: $10 \%$ de fosfato de rocha, $30 \%$ de areia, $30 \%$ de solo argiloso e $30 \%$ de composto orgânico ou esterco curtido. Essa composição era formulada na produção de todas as mudas de espécies arbóreas, produzidas no campo experimental da Embrapa Agrobioogia. Com o passar do tempo, devido a alguns resultados satisfatórios do uso de composto orgânico em outras atividades paralelas de pesquisas no campo experimental, houve uma certa empolgação subjetiva em aumentar a composição volumétrica do teor do composto que chegou a casa dos $70 \%$. Visualmente esse incremento não correspondeu, para as mudas de leguminosas fixadoras de nitrogênio, a um aumento de qualidade das mesmas e, em algumas espécies, foi observado exatamente o oposto. 
Diante de tal fato e pela necessidade de sair da esfera especulativa e entrar na experimentação científica, objetivando dar subsídios à manutenção das recomendações contidas no comunicado técnico e na produção de mudas do centro, foi realizado um ensaio em casa-devegetação, com os substratos em questão, utilizando o sabiá (Mimosa caesalpiniaefolia Benth.) espécie de rápido estabelecimento e crescimento em campo - que é uma das espécies arbóreas mais usadas no Programa de Recuperação de Áreas Degradadas da Embrapa Agrobiologia.

\section{METODOLOGIA}

O experimento foi conduzido em casa-de-vegetação na Embrapa Agrobiologia, no município de Seropédica, RJ, em bandejas de isopor comercial de células para a produção de mudas com 11,5 cm de altura e volume de cada célula de $100 \mathrm{ml}$. Foram utilizados dois substratos para a produção das mudas. O substrato 1 (70-10-10-10) foi constituído de uma mistura contendo: 700 g. $\mathrm{dm}^{-3}$ de composto orgânico; $100 \mathrm{~g} . \mathrm{dm}^{-3}$ de solo argiloso; $100 \mathrm{~g} . \mathrm{dm}^{-3}$ de areia e $100 \mathrm{~g} . \mathrm{dm}^{-3} \mathrm{de}$ fosfato de rocha. O substrato 2 (30-30-30-10) foi constituído de uma mistura contendo: $300 \mathrm{~g} . \mathrm{dm}^{-}$ ${ }^{3}$ de composto orgânico; $300 \mathrm{~g} . \mathrm{dm}^{-3}$ de solo argiloso; $300 \mathrm{~g} \cdot \mathrm{dm}^{-3}$ de areia e $100 \mathrm{~g} . \mathrm{dm}^{-3}$ de fosfato de rocha. A fonte de fósforo adicionada nesses substratos foi o fosfato natural de Araxá (24\% de $\mathrm{P}_{2} \mathrm{O}_{5}$ solúvel em ácido cítrico $2 \%$ - indicação do fabricante). As determinações químicas dos substratos foram realizadas segundo metodologias da EMPRESA BRASILEIRA DE PESQUISA AGROPECUÁRIA - Embrapa (1979), e revelaram pH em água $(1: 2,5)=6,8$ e 6,4; P disponível (resina) $=874$ e $192 \mathrm{mg} \cdot \mathrm{dm}^{-3} ; \mathrm{K}=25,5$ e 12,0 mmol $\cdot \mathrm{dm}^{-3} ; \mathrm{Ca}=477$ e $277 \mathrm{mmol}_{\mathrm{c}} \cdot \mathrm{dm}^{-3} ; \mathrm{Mg}=135$ e 44 $\mathrm{mmol}_{\mathrm{c}} \cdot \mathrm{dm}^{-3} ; \mathrm{H}+\mathrm{Al}^{+++}=160$ e $150 \mathrm{mmol}_{\mathrm{c}} \cdot \mathrm{dm}^{-3} ; \mathrm{CTC}=653$ e $348 \mathrm{mmol}_{\mathrm{c}} \cdot \mathrm{dm}^{-3}$; Matéria Orgânica $=94$ e $68 \mathrm{~g} \cdot \mathrm{dm}^{3}$, respectivamente para os substratos 1 e 2 . Os substratos foram fumigados separadamente em caixas de alvenaria vedadas, com aplicação de $80 \mathrm{~cm}^{3}$ de bromex (Brometo de metila $98 \%$ + cloropicrina $2 \%$ em peso) por $\mathrm{m}^{3}$ de substrato, durante 96 horas.

O delineamento experimental utilizado foi inteiramente casualizado com os tratamentos dispostos em esquema fatorial completo $4 \times 2$ com 5 repetições. Os tratamentos foram: Testemunha sem inoculação (Test.), Inoculado com bradyrhizobium (R); Inoculado com fungo micorrízico arbuscular (M); Inoculado com bradyrhizobium e fungo micorrízico arbuscular (R+M) e os dois substratos para a produção de mudas descritos anteriormente.

Os fungos micorrízicos utilizados como inóculos foram Glomus clarum e Gisgaspora margarita Becher \& Hall, oriundos da Universidade Federal de Lavras - UFLA e mantidos na coleçãoo da Embrapa Agrobiologia e as bactérias foram as estirpes de bradyhizobium selecionadas BR 3405 e BR 3451 oriundas também das coleções da Embrapa Agrobiologia.

Visando a quebra de suas dormências, as sementes de sabiá foram escarificadas com $\mathrm{H}_{2} \mathrm{SO}_{4}$ concentrado durante 3 minutos e logo após lavadas com água destilada. 0 método promoveu um bom índice de germinação. As sementes foram provenientes do campo experimental da Embrapa Agrobiologia e foram coletadas no mesmo ano do plantio do experimento.

Foram colocadas 3 sementes por célula nas bandejas de produção de mudas e, nos tratamentos que receberam inoculações com rizóbios, foram utilizadas as estirpes previamente selecionadas, crescidas em meio semissólido descrito por VINCENT (1970), tendo como veículo turfa esterilizada. Nos tratamentos que receberam inoculações com fungos micorrízicos, foi colocada uma mistura em solução contendo 20 esporos de cada espécie por célula (OLIVEIRA, 
1993). Os inóculos foram produzidos em cultura estoque de Brachiaria decumbens. Os inóculos tanto dos rizóbios como dos fungos foram colocados em cavidades a $2 \mathrm{~cm}$ de profundidade, abertas nos substratos para posterior colocação e cobertura das sementes de acácia. Quando as plântulas apresentavam um par de folhas definitivas, foi realizado o desbaste, deixando uma planta por célula.

O sabiá foi colhido aos 79 dias após o plantio. Os parâmetros avaliados foram altura, massa seca da parte aérea e das raízes, diâmetro à altura do colo, número e peso dos nódulos secos, porcentagem de colonização pelas MVAs e concentração de $\mathrm{N}, \mathrm{P}, \mathrm{K}, \mathrm{Ca}, \mathrm{Mg}, \mathrm{S}, \mathrm{Cu}, \mathrm{Mn}$, Fe e Zn.

O material da parte aérea foi seco a $65^{\circ} \mathrm{C}$, em estufa de circulação forçada de ar e pesado, após secagem, até o atingimento de peso constante. Foi então moído e realizado uma digestão ácida para análises de nitrogênio que foi feita empregando-se a destilação por arraste de vapor (método Kjeldahl). Também no material da parte aérea seca e moída foi feita uma digestão nitroperclórica para a extração simultânea dos demais elementos analisados. A determinação do fósforo foi feita por colorometria; o potássio por fotometria de chama; e o cálcio, magnésio, cobre, manganês, ferro e zinco por espectrofotometria de absorção atômica, segundo as metodologias descritas por BATAGLIA et al. (1983).

Para a verificação da colonização micorrízica, o sistema radicular foi lavado em água destilada e em seguida colocado em papel absorvente para ser retirado o excesso de umidade. Foi retirado $0,5 \mathrm{~g}$ de raízes finas ( $<1 \mathrm{~mm}$ de diâmetro) localizado junto ao substrato onde as mudas foram produzidas e conservados em álcool 70\%. O clareamento com KOH $10 \%$ e a coloração das raízes com azul de tripano, seguiram a metodologia descrita por PHILLIPS \& HAYMAN (1970), adaptados por ABBOTT \& ROBSON (1981).

Os segmentos de raízes frescas $(0,5 \mathrm{~g})$, foram lavados inicialmente em água destilada visando retirar o excesso de álcool no qual estavam conservadas as amostras. Em seguida, as raízes foram aquecidas em $\mathrm{KOH} 10 \%$ a $90^{\circ} \mathrm{C}$ por uma hora em banho-maria, a fim de remover o conteúdo citoplasmático. As raízes após este procedimento, foram novamente lavadas com água destilada e colocadas em água oxigenada a 3\% por 15 minutos. Depois as raízes foram colocadas em $\mathrm{HCL}$ a $2 \%$ por 5 minutos, a fim de retirar os resíduos do $\mathrm{KOH}$. Novamente foram lavadas com água destilada e colocadas em solução de lactoglicerol com 0,05\% de azul de tripano sendo novamente submetidas a banho-maria por 30 minutos. Após estes procedimentos, as raízes foram observadas em microscópio estereoscópico usando uma lente de aumento 40X.

A porcentagem do comprimento das raízes colonizadas foi avaliada pelo método de intersecção em placa, descrito no trabalho de GIOVANETTI \& MOSSE (1980), adaptado a partir do método de medida de comprimento de raízes de NEWMAN (1966).

Os parâmetros estudados foram submetidos às análises de variância e teste de médias, utilizando-se o Sistema de Análises Estatísticas e Genéticas (SAEG), desenvolvido pelo setor de processamento de dados da Universidade Federal de Viçosa (UFV). Todos os parâmetros estudados foram submetidos, antes das análises estatísticas paramétricas usuais, ao teste de Lillieforms (que é uma derivação do teste de Kolmogorov-Smirnov), para verificar se os valores de dados de uma determinada variável seguem ou não uma distribuição de médias e desvios-padrão calculados na mesma amostra (se eles têm distribuições normais). Os dados também foram submetidos ao teste de Cochran que é usado para a verificação da homogeneidade de variâncias. Estes testes visam viabilizar a aplicação das análises de variância, que só poderá ser aplicada a um conjunto de 
observações se estiverem satisfeitas as pressuposições de independência, normalidade e variância constante (VIEIRA \& HOFFMANN, 1989).

\section{RESULTADOS E DISCUSSÕES}

Os efeitos dos substratos utilizados para a produção de mudas, submetidas a diferentes inoculações com rizóbios e fungos micorrízicos arbusculares nos parâmetros de crescimento, nodulação, colonização micorrízica e parâmetros nutricionais (teores e acúmulos de macro e micronutrientes na parte aérea) encontram-se nas tabelas de 1 a 3.

Tabela 1: Médias e testes de médias dos parâmetros de crescimento, nodulação e colonização micorrízica observadas em mudas de sabiá (Mimosa caesalpiniaefolia Benth), submetidas a inoculações com Rizóbio (R) e fungos micorrízicos arbusculares (M), em diferentes substratos, aos 79 dias após o plantio (média de 5 repetições, sendo a unidade experimental composta por 3 plantas - dados expressos por planta).

\begin{tabular}{|c|c|c|c|c|c|c|c|c|c|c|c|}
\hline \multirow{3}{*}{ Parâmetro } & \multirow{3}{*}{$\mathbf{S}$} & \multicolumn{10}{|c|}{ Tratamentos microbiológicos ${ }^{(1)}$} \\
\hline & & \multirow{2}{*}{\multicolumn{2}{|c|}{ Test }} & \multirow{2}{*}{\multicolumn{2}{|c|}{$\mathbf{R}$}} & \multirow{2}{*}{\multicolumn{2}{|c|}{ M }} & \multirow{2}{*}{\multicolumn{2}{|c|}{$\mathrm{R}+\mathrm{M}$}} & \multicolumn{2}{|c|}{ Média } \\
\hline & & & & & & & & & & & \\
\hline \multirow[t]{3}{*}{ Altura - H (cm) } & 1 & 17,92 & $A b$ & 24,87 & $\mathrm{Ba}$ & 17,59 & $\mathrm{Ab}$ & 24,35 & $\mathrm{Ba}$ & 21,19 & \\
\hline & 2 & 20,63 & $\mathrm{Ab}$ & 31,43 & $\mathrm{Aa}$ & 19,84 & $\mathrm{Ab}$ & 31,01 & $\mathrm{Aa}$ & 25,73 & \\
\hline & & 19,29 & & 28,15 & & 18,72 & & 27,87 & & & \\
\hline \multirow{3}{*}{$\begin{array}{l}\text { Diâmetro à Altura } \\
\text { do Colo - D (mm) }\end{array}$} & 1 & 2,03 & & 2,54 & & 2,06 & & 2,57 & & 2,30 & \\
\hline & 2 & 2,05 & & 2,73 & & 1,97 & & 2,70 & & 2,36 & \\
\hline & & 2,04 & $\mathrm{~b}$ & 2,63 & $a$ & 2,01 & $\mathrm{~b}$ & 2,64 & $a$ & & \\
\hline \multirow{3}{*}{$\begin{array}{l}\text { Massa Seca da Parte } \\
\text { Aérea- MSPA(g.pl-1) }\end{array}$} & 1 & 0,66 & & 1,02 & & 0,65 & & 1,04 & & 0,84 & \\
\hline & 2 & 0,75 & & 1,13 & & 0,74 & & 1,11 & & 0,93 & \\
\hline & & 0,71 & $b$ & 1,08 & $a$ & 0,70 & $b$ & 1,01 & $a$ & & \\
\hline \multirow{3}{*}{$\begin{array}{l}\text { Massa Seca das } \\
\text { Raízes - MSR (g.pl }{ }^{-1} \text { ) }\end{array}$} & 1 & 0,32 & & 0,37 & & 0,33 & & 0,36 & & 0,35 & \\
\hline & 2 & 0,33 & & 0,36 & & 0,34 & & 0,36 & & 0,35 & \\
\hline & & 0,33 & $b$ & 0,37 & $a$ & 0,34 & $a b$ & 0,36 & $a b$ & & \\
\hline \multirow{3}{*}{$\begin{array}{l}\text { Massa dos Nódulos } \\
\text { Secos-MNS }\left(\mathrm{g} \cdot \mathrm{pl}^{-1}\right)^{(2)}\end{array}$} & 1 & 0,00 & & 0,05 & & 0,00 & & 0,05 & & 24,90 & $B$ \\
\hline & 2 & 0,00 & & 0,08 & & 0,00 & & 0,08 & & 38,95 & $A$ \\
\hline & & 0,00 & $b$ & 0,06 & $a$ & 0,00 & $b$ & 0,06 & $\mathrm{a}$ & & \\
\hline \multirow{3}{*}{$\begin{array}{l}\mathrm{N}^{0} \text { de Nódulos } \\
\mathrm{NN}^{(2)}\end{array}$} & 1 & 0,00 & $A b$ & 38,13 & $\mathrm{Ba}$ & 0,00 & $A b$ & 44,80 & $\mathrm{Aa}$ & 20,73 & \\
\hline & 2 & 0,00 & $A b$ & 63,73 & $\mathrm{Aa}$ & 0,00 & $\mathrm{Ab}$ & 54,46 & $\mathrm{Aa}$ & 29,55 & \\
\hline & & 0,00 & & 50,93 & & 0,00 & & 49,63 & & & \\
\hline \multirow[t]{3}{*}{ MSR/MSPA } & 1 & 0,50 & & 0,37 & & 0,52 & & 0,35 & & 0,44 & A \\
\hline & 2 & 0,44 & & 0,33 & & 0,46 & & 0,34 & & 0,39 & B \\
\hline & & 0,47 & $a$ & 0,35 & $b$ & 0,49 & $a$ & 0,34 & $b$ & & \\
\hline \multirow[t]{3}{*}{ MMS/NN } & 1 & 0,00 & & 0,001 & & 0,00 & & 0,001 & & 0,001 & \\
\hline & 2 & 0,00 & & 0,001 & & 0,00 & & 0,002 & & 0,001 & \\
\hline & & 0,00 & $b$ & 0,001 & $a$ & 0,00 & $b$ & 0,001 & $a$ & & \\
\hline \multirow{3}{*}{$\begin{array}{l}\text { Taxa de Colonização } \\
\text { Micorrízica }^{(3)}(\%)\end{array}$} & 1 & 0,00 & & 0,00 & & 24.01 & & 23,88 & & 11,97 & \\
\hline & 2 & 0,00 & & 0,00 & & 23,62 & & 24,92 & & 12,13 & \\
\hline & & 0,00 & $b$ & 0,00 & $b$ & 23,81 & $a$ & 24,40 & $a$ & & \\
\hline
\end{tabular}

${ }^{(1)}$ Médias seguidas de mesma letra (maiúscula, compara substratos; minúscula, compara tratamentos microbiológicos) não diferem entre si ao nível de $5 \%$ de probabilidade pelo teste de Tukey.

(2) Dados originais, sendo a análise de variância feita com os dados transformados em $\sqrt{x}+1$.

(3) Dados originais, sendo a análise de variância feita com os dados transformados em arco seno $\sqrt{x} / 100$.

Os tratamentos microbiológicos (TM) isoladamente aumentaram significativamente todos os parâmetros estudados (Tabela 1), ao nível de $1 \%$ de significância do teste $\mathrm{F}$. Em relação à 
testemunha, os incrementos médios (em percentual), apresentados na sequência ( $R, M$ e $R+M)$ dos parâmetros de crescimento, foram respectivamente os seguintes: (46, -3 e 44\%) para a altura (H); (29, -1 e 29\%) para o diâmetro (D); (46, -1,5 e 44\%) para a massa seca da parte aérea (MSPA) e (12, 3 e 12\%) para a massa seca das raízes (MSR). O substratos (S) isoladamente, só tiveram efeitos significativos nos parâmetros: altura $(H)$, massa dos nódulos secos (MNS), número de nódulos (NN) e na relação massa seca das raízes/massa seca da parte aérea (MSR/MSPA) nos dois níveis de significância (1 e 5\%) do teste F. Somente ocorreu interação significativa (TM X S) nos parâmetros altura(H) e número de nódulos (NN).

Com exceção da massa seca das raízes e a relação massa seca das raízes/massa seca da parte aérea, os tratamentos com rizóbio isolado ou em dupla inoculação, foram superiores em todos os parâmetros de crescimento analisados.

O substrato 2, em média, foi superior no parâmetro massa dos nódulos secos e o substrato 1 na relação MSR/MSPA. Na interação significativa (TM X S) o número de nódulos no substrato 2 foi superior no tratamento com dupla inoculação microbiológica, ao passo que no parâmetro altura das planta, os tratamentos microbiológicos que utilizaram rizóbios em sua composição no substrato 2 , foram superiores ao substrato 1 .

A dupla inoculação ( $R+M)$ não aumentou a taxa de colonização micorrízica no sabiá. Também não houve colonização por este simbionte nos tratamentos não inoculados com o mesmo. Segundo vários autores, a dupla inoculação é um procedimento vantajoso para o crescimento de muitas espécies arbóreas favorecendo a fixação de N, a produção de biomassa e o estabelecimento das plantas no campo (TAVARES, 1998). Esta vantagem, entretanto, só se verifica quando existe uma compatibilidade entre os simbiontes. Enquanto algumas estirpes aumentam a produção de massa seca na parte aérea na presença de fungos micorrízicos arbusculares, outras, ditas incompatíveis, são capazes de aumentar a produção de massa seca quando inoculadas isoladamente, mas a mesma produção não é atingida quando essas estirpes são co-inoculadas com fungos micorrízicos (LEITÃO, 1997).

A taxa de colonização média dos tratamentos submetidos as inoculações fúngicas ficou na casa dos $25 \%$, que para o sabiá, embora seja considerada baixa, está compatível com os resultados de outros autores que trabalharam com sistemas de produção de mudas da espécie estudada semelhantes a este ensaio (MONTEIRO, 1990 e OLIVEIRA, 1993).

A comparação de valores absolutos de taxa de colonização em diferentes trabalhos deve ser feita com precaução, porque alguns métodos podem superestimar esses valores e também pelo fato de inúmeros fatores atuarem na determinação dessas taxas, com: diferentes substratos utilizados, diferentes quantidades efetivas de inoculantes, diferentes isolados, diferentes sementes e procedências, etc. O que interessa na realidade, é que a inoculação cumpra os seus objetivos (principalmente promovendo aumentos na produção de matéria seca), o que não significa que em baixos níveis isso não possa ocorrer. 
Tabela 2: Médias e testes de médias dos parâmetros nutricionais (teores e acúmulo de macronutrientes na parte aérea), observadas em mudas de sabiá (Mimosa caesalpiniaefolia Benth), submetidas a inoculações com Rizóbio

(R) e fungos micorrízicos arbusculares (M), em diferentes substratos, aos 79 dias após o plantio (média de 5 repetições, sendo a unidade experimental composta por 3 plantas - dados expressos por planta).

\begin{tabular}{|c|c|c|c|c|c|c|c|c|c|c|c|}
\hline \multirow{3}{*}{ Parâmetro } & \multirow{3}{*}{$\mathbf{S}$} & \multicolumn{10}{|c|}{ Tratamentos microbiológicos ${ }^{(1)}$} \\
\hline & & \multirow{2}{*}{\multicolumn{2}{|c|}{ Test }} & \multirow{2}{*}{\multicolumn{2}{|c|}{$\mathbf{R}$}} & \multirow{2}{*}{\multicolumn{2}{|c|}{ M }} & \multirow{2}{*}{\multicolumn{2}{|c|}{$\mathbf{R}+\mathbf{M}$}} & \multicolumn{2}{|c|}{ Média } \\
\hline & & & & & & & & & & & \\
\hline \multirow[t]{3}{*}{$\mathrm{N}\left(\mathrm{g} \cdot \mathrm{Kg}^{-1}\right)$} & 1 & 19,93 & & 32,12 & & 19,77 & & 31,15 & & 25,74 & \\
\hline & 2 & 19,81 & & 36,04 & & 19,84 & & 33,84 & & 27,38 & \\
\hline & média & 19,87 & $\mathrm{~b}$ & 34,08 & a & 19,81 & $\mathrm{~b}$ & 32,49 & a & & \\
\hline \multirow[t]{3}{*}{ N Total (mg/planta) } & 1 & 13,08 & & 32,99 & & 12,99 & & 32,83 & & 22,98 & \\
\hline & 2 & 15,07 & & 41,01 & & 14,74 & & 37,55 & & 27,09 & \\
\hline & média & 14,08 & $\mathrm{~b}$ & 37,07 & a & 13,86 & $\mathrm{~b}$ & 35,19 & a & & \\
\hline \multirow[t]{3}{*}{$\mathrm{P}\left(\mathrm{g} \cdot \mathrm{Kg}^{-1}\right)$} & 1 & 2,64 & & 1,82 & & 2,40 & & 1,76 & & 2,15 & A \\
\hline & 2 & 2,36 & & 1,78 & & 2,18 & & 1,74 & & 2,02 & B \\
\hline & média & 2,50 & $a$ & 1,80 & $b$ & 2,29 & $a$ & 1,75 & $\mathrm{~b}$ & & \\
\hline \multirow[t]{3}{*}{ P Total (mg/planta) } & 1 & 1,74 & & 1,86 & & 1,56 & & 1,84 & & 1,75 & \\
\hline & 2 & 1,78 & & 2,01 & & 1,59 & & 1,94 & & 1,83 & \\
\hline & média & 1,76 & & 1,94 & & 1,58 & & 1,89 & & & \\
\hline \multirow[t]{3}{*}{$\mathrm{K}\left(\mathrm{g} \cdot \mathrm{Kg}^{-1}\right)$} & 1 & 16,14 & & 16,30 & & 16,52 & & 16,38 & & 16,33 & \\
\hline & 2 & 15,88 & & 15,54 & & 16,24 & & 14,72 & & 15,59 & \\
\hline & média & 16,01 & & 15,92 & & 16,38 & & 15,55 & & & \\
\hline \multirow[t]{3}{*}{ K Total (mg/planta) } & 1 & 10,59 & & 16,63 & & 10,83 & & 16,95 & & 13,75 & \\
\hline & 2 & 11,86 & & 17,65 & & 11,92 & & 16,23 & & 14,42 & \\
\hline & média & 11,22 & $\mathrm{~b}$ & 17,14 & $a$ & 11,37 & $\mathrm{~b}$ & 16,59 & $a$ & & \\
\hline \multirow[t]{3}{*}{ Ca $\left(\mathrm{g} . \mathrm{Kg}^{-1}\right)$} & 1 & 14,26 & $\mathrm{Aa}$ & 14,16 & $\mathrm{Aa}$ & 17,10 & $\mathrm{Aa}$ & 13,90 & Aa & 14,85 & \\
\hline & 2 & 15,88 & $\mathrm{Aa}$ & 9,92 & $\mathrm{Bb}$ & 16,88 & $\mathrm{Aa}$ & 8,84 & $\mathrm{Bb}$ & 12,88 & \\
\hline & média & 15,07 & & 12,04 & & 16,99 & & 11,37 & & & \\
\hline \multirow[t]{3}{*}{ Ca Total (mg/planta) } & 1 & 9,42 & $\mathrm{Ab}$ & 14,36 & $\mathrm{Aa}$ & 10,98 & Aab & 14,49 & Aa & 12,31 & \\
\hline & 2 & 11,61 & $\mathrm{Aa}$ & 11,08 & $\mathrm{Ba}$ & 12,33 & $\mathrm{Aa}$ & 9,75 & $\mathrm{Ba}$ & 11,19 & \\
\hline & média & 10,52 & & 12,72 & & 11,66 & & 12,12 & & & \\
\hline \multirow[t]{3}{*}{$\mathrm{Mg}\left(\mathrm{g} \cdot \mathrm{Kg}^{-1}\right)$} & 1 & 3,21 & & 3,57 & & 3,45 & & 3,62 & & 3,46 & A \\
\hline & 2 & 2,84 & & 2,47 & & 3,26 & & 2,71 & & 2,82 & B \\
\hline & média & 3,02 & & 3,02 & & 3,35 & & 3,16 & & & \\
\hline \multirow[t]{3}{*}{ Mg Total(mg/planta) } & 1 & 2,11 & & 3,58 & & 2,26 & & 3,78 & & 2,94 & \\
\hline & 2 & 2,13 & & 2,80 & & 2,40 & & 3,00 & & 2,58 & \\
\hline & média & 2,12 & $\mathrm{~b}$ & 3,19 & $a$ & 2,33 & $\mathrm{~b}$ & 3,39 & $a$ & & \\
\hline
\end{tabular}

Médias seguidas de mesma letra (maiúscula, compara substratos; minúscula, compara tratamentos microbiológicos) não diferem entre si ao nível de $5 \%$ de probabilidade pelo teste de Tukey.

De uma maneira geral os diversos tratamentos tiveram mais efeitos nas concentrações dos macronutrientes do que nas concentrações dos micronutrientes, bem como nos acúmulos dos diversos nutrientes pelo sabiá (Tabelas 2 e 3).

Os tratamentos microbiológicos isoladamente tiveram efeitos significativos nos teores de $\mathrm{N}, \mathrm{P}$, e Ca e não surtiram efeitos em $\mathrm{K}$ e $\mathrm{Mg}$. Com relação aos teores de micronutrientes somente o Zn foi afetado pelas inoculações biológicas. As variações médias (em percentual) em relação à testemunha, apresentada na sequência $(R, M$ e $R+M)$ no total acumulado de macro e micronutrientes, foram respectivamente: $(163,-2$ e $149 \%)$ para o $\mathrm{N} ;(10,-10$ e $7 \%)$ para o $\mathrm{P}$; (53, 1 e $48 \%$ ) para o K; (21, 11 e 15\%) para o Ca; (50, 10 e 60\%) para o $\mathrm{Mg}$; (56, 9 e 51\%) para o Cu; (63, 8 e $43 \%)$ para o $\mathrm{Mn} ;(41,-16$ e $35 \%)$ para o Fe e (11, 5 e $12 \%)$ para o $\mathrm{Zn}$. Os substratos isoladamente 
foram significativos nos parâmetros $\mathrm{P}, \mathrm{Ca}, \mathrm{Mg}, \mathrm{CuT}, \mathrm{Mn}$ e MnT. Somente houve interação significativa no teor e acúmulo de Ca. No geral, os dados relativos aos nutrientes (com exceção do Ca), confirmam a falta de resposta do sabiá à adição dos fungos micorrízicos.

Devido a baixa dependência micorrízica (DM) do sabiá demonstrado nesta pesquisa, praticamente houve pouco incremento nos acúmulos dos nutrientes em geral (Tabelas 2 e 3). Quando ocorreu diferenciação entre os tratamentos microbiológicos este se deu somente quando a micorriza foi inoculada conjuntamente com o rizóbio (R+M). MILAGRES E BORGES (1997), trabalhando com fungos micorrízicos Glomus mosseae e Glomus etunicatum e duas estirpes de rizóbio selecionadas para Anadenanthera peregrina e Dalbergia nigra, em solo fumigado, observaram que a inoculação com fungos micorrízicos aumentou significativamente o crescimento das mudas das duas espécies. No entanto, a inoculação com rizóbio somente foi eficiente na presença dos fungos, quando ao grau de dependência micorrízica foi baixo. Segundo esses autores, a dependência micorrízica exerce efeito na simbiose fungo-planta, em que no referido trabalho foi observado que quanto maior o grau de dependência micorrízica manor o efeito da inoculação com rizóbio.

Os teores de $\mathrm{P}, \mathrm{K}, \mathrm{Ca}, \mathrm{Mg}$ e $\mathrm{Zn}$ foram menores nos tratamentos envolvendo rizóbio em relação aos tratamentos sem bactérias, devido a efeitos de diluição no tecido vegetal provocado por um maior acúmulo da massa seca nestes tratamentos das mudas.

Nos parâmetros nutricionais onde o substrato 1 foi estatisticamente superior ao substrato 2 (concentrações de $\mathrm{P}$ e $\mathrm{Mg}$ ), na prática se anulam no acúmulo total destes nutrientes, que não apresentam diferenças estatísticas ao limite de $5 \%$ estabelecido pelos testes. No caso inverso, a maior concentração de $\mathrm{Mn}$ no substrato 2 é acompanhada também pelo maior acúmulo deste nutriente nos tecidos foliares, onde esse elemento exprime sua mais importante função, que é participar de processos de oxi-redução da planta (DECHEN et al., 1991).

Neste ensaio, foram observados que os tratamentos microbiológicos ( $R$ e $R+M)$, nos dois substratos esterilizados, quando comparados com o controle sem inoculação e inoculados somente com os fungos micorrízicos (M), favoreceram o desenvolvimento das mudas em quase todos os parâmetros avaliados (Tabelas 1 a 3).

MENDES, 2010 avaliou o crescimento do Sabiá em resposta também à inoculação com Rizóbio e Fungos Micorrízicos Arbusculares e demonstrou que a inoculação conjunta de Glomus clarum + Gigaspora margarita + BR3405 teve a melhor performance simbiótica, proporcionando os maiores valores de crescimento, tanto em altura como em diâmetro do colo, para as plantas de Sabiá.

SOUZA et al., 2009 avaliaram a influência de dois tipos de substrato (S1 e S2), na ausência e na presença de inoculação de fungos micorrízicos arbusculares (FMA), na produção de mudas de Schinus terebinthifolius em tubetes de $280 \mathrm{~mL}$. S1 e S2 se constituíram numa mistura (em volume), previamente desinfestada, de fosfato de rocha (10\%), areia grossa lavada (30\%), material argiloso (30\%) e matéria orgânica (30\%). Para S1, a fonte de matéria orgânica foi resíduo industrial de produção de borracha sintética, enquanto que para S2, esterco bovino curtido. Aos 120 dias após a montagem do experimento, observou-se que $\mathrm{S} 2$ proporcionou às mudas ganhos significativos em praticamente todas as variáveis analisadas (altura, diâmetro, massa seca de parte aérea e de raiz, teor de N, P e K na parte aérea, e de $\mathrm{N}$ na raiz), em comparação com S1. Não houve diferença significativa entre os substratos quanto à ausência e presença de FMA, possivelmente em função 
dos elevados teores de nutrientes em S1 e S2. A esse mesmo fato se atribuiu a ausência de colonização de raízes por FMA em S2. Portanto, S2 mostrou ser mais indicado para a produção de mudas de $S$. terebinthifolius.

As plantas inoculadas somente com os fungos micorrízicos não superaram (em praticamente todos os parâmetros) as plantas controle, mostrando desta maneira a falta de resposta à adição de MA nestas condições experimentais.

Tabela 3: Médias e testes de médias dos parâmetros nutricionais (teores e acúmulo de micronutrientes na parte aérea), observadas em mudas de sabiá (Mimosa caesalpiniaefolia Benth), submetidas a inoculações com Rizóbio

(R) e funos micorrízicos arbusculares (M), em diferentes substratos, aos 79 dias após o plantio (média de 5 repetições, sendo a unidade experimental composta por 3 plantas - dados expressos por planta).

\begin{tabular}{|c|c|c|c|c|c|c|c|c|c|c|c|}
\hline & & & & & rat & nentos $n$ & crol & $\operatorname{lógicos}^{(1)}$ & & & \\
\hline Parâmetro & $\mathbf{S}$ & & & & & & & & & Méd & \\
\hline & & Tes & & $\mathbf{R}$ & & $\mathbf{M}$ & & $\mathrm{R}+\mathrm{M}$ & & & \\
\hline $\mathrm{Cu}\left(\mathrm{mg} . \mathrm{Kg}^{-1}\right)$ & 1 & 10,00 & & 10,00 & & 10,60 & & 10,40 & & 10,25 & \\
\hline & 2 & 0,00 & & 10,80 & & 11,60 & & 10,00 & & 10,60 & \\
\hline & media & 10,00 & & 10,40 & & 11,10 & & 10,20 & & & \\
\hline Cu Total ( $\mu \mathrm{g} /$ planta) & 1 & 6,63 & & 10,11 & & 6,88 & & 10,83 & & 8,61 & $B$ \\
\hline & 2 & 7,53 & & 11,99 & & 8,55 & & 10,61 & & 9,67 & A \\
\hline & média & 7,08 & $b$ & 11,05 & a & 7,71 & $b$ & 10,72 & $a$ & & \\
\hline $\mathrm{Mn}\left(\mathrm{mg} \cdot \mathrm{Kg}^{-1}\right)$ & 1 & 48,00 & & 52,00 & & 52,00 & & 46,00 & & 49,50 & $B$ \\
\hline & 2 & 58,00 & & 62,00 & & 66,00 & & 58,00 & & 61,00 & A \\
\hline & média & 53,00 & & 57,00 & & 59,00 & & 52,00 & & & \\
\hline Mn Total( $\mu \mathrm{g} /$ planta $)$ & 1 & 31,54 & & 54,37 & & 33,95 & & 46,58 & & 41,61 & $B$ \\
\hline & 2 & 44,39 & & 69,66 & & 47,95 & & 61,83 & & 55,96 & A \\
\hline & média & 37,96 & $\mathrm{C}$ & 62,02 & $a$ & 40.95 & bc & 54,20 & $a b$ & & \\
\hline $\mathrm{Fe}\left(\mathrm{mg} \cdot \mathrm{Kg}^{-1}\right)$ & 1 & 184,0 & & 166,0 & & 174,0 & & 164,0 & & 172,0 & \\
\hline & 2 & 186,0 & & 178,0 & & 146,0 & & 162,0 & & 168,0 & \\
\hline & média & 185,0 & & 172,0 & & 160,0 & & 163,0 & & & \\
\hline Fe Total ( $\mu \mathrm{g} /$ planta) & 1 & 122,5 & & 168,3 & & 113,4 & & 174,0 & & 144,6 & \\
\hline & 2 & 140,8 & & 202,5 & & 108,3 & & 182,9 & & 158,6 & \\
\hline & média & 131,7 & $a b$ & 185,4 & $a$ & 110,6 & $b$ & 178,4 & $a$ & & \\
\hline $\mathrm{Zn}\left(\mathrm{mg} \cdot \mathrm{Kg}^{-1}\right)$ & 1 & 17,40 & & 13,50 & & 19,24 & & 13,88 & & 16,01 & \\
\hline & 2 & 20,04 & & 14,44 & & 20,45 & & 14,32 & & 17,31 & \\
\hline & média & 18,72 & $a b$ & 13,97 & $b$ & 19,84 & $a$ & 14,10 & $b$ & & \\
\hline Zn Total ( $\mu \mathrm{g} /$ planta) & 1 & 11,55 & & 13,48 & & 12,74 & & 14,43 & & 13,05 & \\
\hline & 2 & 15,15 & & 16,21 & & 15,41 & & 15,56 & & 15,58 & \\
\hline & média & 13,35 & & 14,84 & & 14.08 & & 14,99 & & & \\
\hline
\end{tabular}

(1)Médias seguidas de mesma letra (maiúscula, compara substratos; minúscula, compara tratamentos microbiológicos) não diferem entre si ao nível de $5 \%$ de probabilidade pelo teste de Tukey.

Os teores dos micronutrientes ( $\mathrm{Cu}, \mathrm{Mn}$ e $\mathrm{Fe}$ ) não diferiram entre os tratamentos, somente ocorrendo aumento no teor de $\mathrm{Zn}$ nos tratamentos testemunha e inoculado somente com rizóbio. Ao passo que suas acumulações foram maiores no tratamento $\mathrm{M}$ e $\mathrm{R}+\mathrm{M}$.

De uma maneira geral, na produção de mudas de Mimosa caesalpiniaefolia, verificou-se nas tabelas apresentadas que são poucos os parâmetros onde o substrato 1 apresenta superioridade em relação ao substrato 2 . Logo, por questão de economia e pelos resultados obtidos nos parâmetros morfológicos e nutricionais das mudas, deve-se optar pelo substrato 2 . 


\section{CONCLUSÃO}

Em relação aos substratos esterilizados, utilizados para a produção de mudas e inoculados com os tratamentos microbiológicos em que foi desenvolvido o experimento, pode-se chegar às conclusões seguintes:

- Na Mimosa caesalpiniaefolia Benth, as inoculações com rizóbios isolados (R) ou em conjunto com os fungos micorrízicos (R+M), beneficiam a formação das mudas do sabiá em todos parâmetros de crescimento estudados em relação aos outros tratamentos microbiológicos (Testemunha e M); aumentaram os teores de $\mathrm{N}$ e o acúmulo de $\mathrm{N}, \mathrm{K}, \mathrm{Mg}, \mathrm{Cu}, \mathrm{Mn}$ e Fe;

- A inoculação com micorriza estimulou a nodulação das mudas;

- O substrato 2 foi superior ao substrato 1 em dois importantes parâmetros quantitativos em relação a mudas de sabiá a serem utilizadas para o plantio de campo: Massa dos nódulos secos; e acúmulo de $\mathrm{Cu}$ e $\mathrm{Mn}$ Total e na concentração de $\mathrm{Mn}$, além do mais, em praticamente nenhum parâmetro analisado foi estatisticamente inferior, somente o sendo nas concentrações de $\mathrm{P}$ e $\mathrm{Mg}$ total;

- Os resultados finais indicam que, em relação as variáveis estudadas no presente trabalho e aos aspectos econômicos e práticos, deve-se na produção de mudas dessas espécies, optar pelo substrato 2 , que possui um menor teor de composto orgânico em sua composição.

\section{REFERÊNCIAS BIBLIOGRÁFICAS}

1. BRAGA, Roberto. Fundamentos e técnicas de administração financeira. 1 ed. São Paulo: Atlas, 1989.

2. BRIGHAM, E. F. et al. Administração financeira: teoria e prática. São Paulo: Atlas, 2001.

3. BATAGLIA, O. C. et al. Métodos de análise química das plantas. Instituto Agronômico, Campinas, 1983. 48 p. (Boletim 78). 2001. CD-ROM.

4. CARNEIRO, J. G. A. Produção e controle de qualidade de mudas florestais. UFPR/FUPEF/UENF, Viçosa, 1995. 451p.

5. CARVALHO O.O. et al. Perfil Industrial da cerâmica vermelha no Rio Grande do Norte. Natal: Federação das Indústrias do Rio Grande do Norte, 2001. CD-ROM.

6. EMPRESA BRASILEIRA DE PESQUISA AGROPECUÁRIA. Revegetação de solos degradados. Itaguaí, 1992. 9p.

7. EMPRESA BRASILEIRA DE PESQUISA AGROPECUÁRIA. Manual de análises de solos. Brasília, 1989. 179p.

8. FONSECA, F. A. Produção de mudas de Acacia mangium e Mimosa artemisiana utilizando resíduos urbanos como substratos, associados a fungos micorrízicos arbusculares., Seropédica: Embrapa Agrobiologia, 2010. 24 p. (Embrapa Agrobiologia. Boletim de Pesquisa e Desenvolvimento, 67).

9. FRANCO. et al. Uso de leguminosas florestais noduladas e micorrizadas como agentes de recuperação e manutenção da vida do solo: Um modelo tecnológico. In: ESTEVES, F. A. De. Estrutura, funcionamento e manejo de ecossistemas brasileiros. S1p., 1995. P 459-467. 
10. GOI, S. R. Nitrogen nutrition of nodulated wood legumes. PhD. Thesis. University of Dundee, Scotland, U.K., 1993.

11. GOI, S. R.; SPRENT, J. I.; JAMES, E. K. \& JACOB-NETO, J. Influence of nitrogen form and concentrations of the nitrogen fixation of Acacia auriculiformis. Symbiossis, V. 14, p.115-122, 1992.

12. GOMES, J. M.; COUTO, L. \& PEREIRA, A. R. Uso de diferentes substratos na produção de mudas de Eucalyptus grandis em tubetes e em bandejas de isopor. Revista Árvore, 9 (1): 58-86, 1985.

13. HABTE, M. \& SOEDARJO, M. Mycorrhizal inoculation efect in Acacia mangium grown in a acid oxisol amended with gypsum. Journal of plant nutrition. 18 (10): 2059-2073., 1995.

14. HUNGRIA, M. Coleta de nódulos e isolamento de rizóbio. In: HUNGRIA, M. \& ARAÚJO<R. S., eds. Manual de métodos empregados em estudos de microbiologia agrícola. EMBRAPA-SPI, Brasília, 1994. P 45-61.

15. MANJUNATH, A.; BAGYARAJ, D. J. \& GOPALA-GOWDA, H. S. Dual inoculation with VA mycorrhiza and Bradyrhizobium is benefical to Leucaena. Plant and Soil., 78:445-448, 1984.

16. MARTINS, M. A. \& READ, D. J. Efeitos da disrupção do micélio externo de fungos micorrízicosarbusculares sobre o crecimento vegetal. Pesquisa Agropecuária Brasileira. Brasília, v. 32, n. 11, p.1183-1189, 1997.

17. MENDES FILHO, P. F. Efeito da interação Bradyrhizobium. Micorrizas VA e fosfatos no desenvolvimento de mudas de sabiá (Mimosa caesalpiniaefolia Benth. Fortaleza, UFC, 1985. 51 p. (Dissertação de mestrado).

18. OLIVEIRA, E. Efeito do número de esporos de fungos micorrízicos arbusculares no desenvolvimento inicial do sabiá (Mimosa caesalpiniaefolia Benth) em solo ácido. Itaguaí, Universidade Federal Rural do Rio de Janeiro, 1993. 173 p. (Dissertação de mestrado).

19. SUN, J.S.; SIMPSON, R.J \& SANDS, R. Nitrogenase activity of two genotypes of Acacia mangium as affected by phosphorus nutrition. Plant and Soil. 144: 51-58, 1992. A.

20. VASCONCELOS, I. ALMEIDA, R. T. De \& NESS, R. L. L. Competição entre 19 estirpes de Bradyrhizobium sp. Em simbiose com sabiá, Mimosa caesalpiniaefolia Benth. Ciência Agronômica., 17(1):99-103, 1986.

21. VIEIRA, S. \& HOFFMANN, R. Estatística Experimental. Ed. Atlas, São Paulo, 1989. 179p.

22. ZHOU, M.; RHUE, R. D. \& HARRIS, W. G. Phosfhorus Sorption Characteristics of Bh and Bt Horizons from Sandy Coastal Plain Soils. Soil. Sci. Soc. Am. J. 61:1364-1369. 1997. 